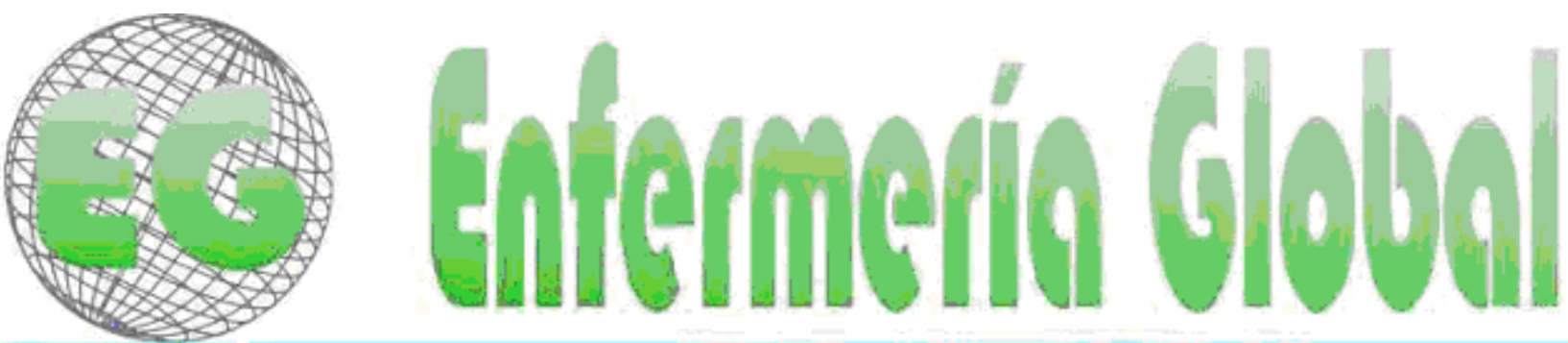

$\mathrm{N}^{\circ} 19$

Revista electrônica cuatrimestral de Enfermeria

Junio 2010

www.um.es/egloball

DOCENCIA - FORMACIÓN

\title{
NIVEL DE ESTRÉS Y ESTRATEGIAS DE AFRONTAMIENTO EN ESTUDIANTES DE LA FACULTAD DE ENFERMERÍA - UNSA AREQUIPA. 2006
}

STRESS LEVEL AND COPING STRATEGIES IN NURSING STUDENTS AT THE UNSA FACULTY, AREQUIPA 2006

\begin{abstract}
"Ticona Benavente, SB., "**Paucar Quispe, G., "**Llerena Callata, G.
*Pesquisadora de la Facultad de Enfermería de la Universidad de São Paulo. Brasil.**Pesquisadora de la Facultad de Enfermería de la Universidad Nacional de San Agustín de Arequipa. Perú. ***Doctora en Enfermería, profesora a tiempo completo de la Facultad de Enfermería de la Universidad Nacional de San Agustín de Arequipa.
\end{abstract}

Palabras clave: Estrés, Estrategias de afrontamiento, Relación, Estudiantes.

Keywords: Stress, Coping Strategies, Relationship, and Students

\section{RESUMEN}

La presente investigación titulada "Nivel de estrés y Estrategias de Afrontamiento en estudiantes de la Facultad de Enfermería-UNSA. Arequipa 2006", tuvo como objetivo determinar la relación entre el nivel de estrés y las estrategias de afrontamiento, para tal efecto se realizó un estudio de tipo transeccional con diseño correlacional, la muestra en estudio fue de 234 estudiantes de primero a cuarto año, seleccionada mediante un muestreo probabilístico estratificado. Para la recolección de información se utilizó como método, la encuesta; técnica el cuestionario y como instrumentos: la Escala de Estrés de Holmes y Rahe y el Cuestionario de estimación de Afrontamiento de COPE, que considera estrategias enfocadas al problema, emoción y percepción. Como conclusiones se obtuvo que: 1) la mayor parte de la población estudiada fue de sexo femenino, con rango de edad de 19 a 20 años, el año de estudio que apuntó mayor número de estudiantes y mayor nivel de estrés fue el primer año, seguido de segundo, tercero y cuarto año; además la gran mayoría de estudiantes registra matrícula regular, 2) El nivel de estrés se encontró severo (29.91\%). 3) Las estrategias más utilizadas orientadas al problema fueron: "planificación" y "afrontamiento activo"; orientadas a la emoción: "reinterpretación positiva y crecimiento" y "negación" y orientadas a la percepción: "desentendimiento mental" y "enfocar y liberar emociones".

Finalmente, se hizo uso de la prueba estadística de correlación del estadístico $\mathrm{F}$ para la comprobación de la hipótesis, concluyendo que existe relación entre el nivel de estrés y las estrategias de afrontamiento. 


\section{ABSTRACT}

The present investigation entitled "Stress Level and Coping Strategies in Nursing Students At The Unsa Faculty, Arequipa 2006," had as its aim to determine the relationship between stress level and coping strategies, to this effect a cross-sectional type study with correlation design was made. The sample under study was 234 students from 1st to 4th year, selected through a grouped probability sampling. For the gathering of information, the survey was used as a method; a questionnaire as the technique, and the Social Readjustment Rating Scale of Holmes and Rahe and the COPE's Questionnaire of Coping Estimation as instruments, which consider problem focused strategies, emotion and perception. The conclusions obtained are: 1) the majority of the studied population was of female gender, with an age range from 19 to 20 years, the year of study noted a higher number of students and higher level of stress the 1st year, followed by $2 \mathrm{nd}$, 3rd and 4th year. The great the majority of students reported regular enrolment, 2) The stress level was severe (29.91\%). 3) The most utilized strategies directed at the problem were "planning" and "active coping," aimed at emotion: "positive reinterpretation and growth" and "denial," and targeted to perception: "mental misunderstanding" and "focusing and release of emotions."

Finally, the statistical test of correlation of $F$ statistic was used for hypothesis verification, and it concluded that there is a relationship between the level of stress and coping strategies.

\section{INTRODUCCIÓN}

Durante el proceso de vida, el ser humano busca adaptarse continuamente a los cambios que le ocurren, tratando de lograr el equilibrio entre su organismo y el medio ambiente lo que origina una respuesta al estrés necesaria para afrontar nuevas situaciones ${ }^{1,2,3}$. Sin embargo, la época contemporánea tan compleja, ocasiona que esta respuesta al estrés, que podría ser normal, es decir, sin posibilidades de presentar manifestaciones psicosomáticas, se torna en una acción altamente perjudicial que afecta la calidad de vida ${ }^{4}$.

Actualmente el estrés y su estudio han alcanzado gran popularidad tanto en el lenguaje cotidiano como en la literatura científica, siendo considerado como un factor predisponente, desencadenante o coadyuvante de múltiples enfermedades, las cuales pueden ser realmente serias $3,4,5,6,7,8,9,10,11$

La institución universitaria ha introducido efectos potencialmente provocadores de estrés, de ellos sin duda uno de los más relevantes, es la competitividad ${ }^{12}$. Son muchos los estudiantes y no tantas las oportunidades del mercado laboral, ello hace que ser "de los mejores" sea un objetivo de los estudiantes; por otro lado, a lo largo de la vida universitaria, los estudiantes están sometidos constantemente a exigencias académicas, exceso de responsabilidad, constantes evaluaciones, exposiciones y realización de trabajos ${ }^{13,14,15,16}$, así como a las presiones familiares, económicas y sociales que sin darse cuenta provocan grado de estrés importante el cual se manifiesta generalmente como resultado de las frustraciones originadas por necesidades insatisfechas.

La tensión originada por el estrés es en cierta medida necesaria para el desarrollo de la personalidad del estudiante; sin embargo, cuando el estrés va más allá de los niveles óptimos agota sus energías, deteriora su desempeño y puede llegar a dañar su salud ${ }^{17,18}$. Así mismo, lo que es considerado estresante para unos no lo es para otros, originando que los estudiantes en un intento de adaptarse a estas situaciones pongan en marcha una serie de estrategias de afrontamiento para superar con éxito las exigencias que se les demanda, buscando el alivio de su estado tensional; sin embargo, una gran parte de estudiantes universitarios carecen de estrategias o adoptan estrategias inadecuadas ${ }^{14}$. 
Los estudiantes universitarios, en particular los de ciencias médicas ${ }^{19,20}$, están frecuentemente imbuidos en situaciones estresantes por lo que se hace necesario conocer a qué niveles de estrés están sometidos y cómo lo enfrentan.

Por esto nos propusimos el presente trabajo, pretendiendo identificar las estrategias de afrontamiento frente a los diferentes niveles de estrés, con el propósito de orientar la proyección de actividades promocionales y de prevención o control de la salud en esta población, así como el de constituir un aporte para futuras investigaciones.

\section{FUNDAMENTACIÓN TEÓRICA}

Según la $\mathrm{OMS}^{21}$, a inicios del presente siglo, las enfermedades provocadas por el estrés habrán superado a las enfermedades infecciosas y se estima que en el año 2010 en América Latina y el Caribe, se tendrán más de 88 millones de personas con trastornos afectivos desencadenados por el estrés, y es que los estresores de la vida originan que las personas se ubiquen en una situación incómoda emocional y somáticamente, debiendo buscar la forma de cómo responder ante sus problemas.

En el Perú la prevalencia anual del estrés es del 14\% aproximadamente, según Sánchez ${ }^{19}$ en el año 2001, siendo más frecuente en mujeres que en varones. El estrés es uno de los factores más importantes para la mayoría de las enfermedades más frecuentes que se manifiestan en nuestro siglo, presentándose tanto trastornos cardiacos, hipertensión arterial, cáncer, alteraciones metabólicas y hormonales ${ }^{21,22}$.

Ahora bien, el grupo de estudiantes que ingresa por primera vez en la institución universitaria, se encuentra con la necesidad de establecer un cambio de perspectiva en su comportamiento para adaptarse al nuevo entorno ${ }^{23}$. Esto, en muchos casos, trae consigo la necesidad de que los estudiantes adopten una serie de estrategias de afrontamiento muy distintas a las que disponían hasta ese momento para superar con éxito las nuevas exigencias que se les demandan. Sin embargo, una gran parte de esos nuevos estudiantes universitarios carecen de esas estrategias o presentan unas conductas académicas que son inadecuadas para las nuevas demandas ${ }^{14}$. Olivares ${ }^{23}$ al investigar sobre "Conductas de afrontamiento al estrés en adolescentes pre-universitarios. Academias Fleming y Bardet. Arequipa", en una muestra de 277 estudiantes, concluyó que los estudiantes presentaron generalmente estrés severo (42.1\%) predominando el sexo masculino, las conductas de afrontamiento que adoptaron en su tiempo libre fueron: escuchar música $(79.5 \%)$, lectura $(58.7 \%)$, la práctica de un deporte $(62.2 \%)$, entre los modelos de consumo los más frecuentes fueron el consumir golosinas azucaradas (85.9\%) y bebidas alcohólicas (39.2\%).

La competitividad actual en la cual los estudiantes son constantemente evaluados para atravesar niveles de educación, provoca estados de tensión y frustración por las exigencias académicas en su preparación, así también como la proximidad y fracaso experimentado en los exámenes, sobrecarga curricular, ausencia de pausas entre clases, exceso de responsabilidad, tiempo limitado para hacer trabajos ${ }^{12}$. En Venezuela, Rodríguez ${ }^{14}$ al investigar sobre los "Efectos de un plan de técnicas de estudio en estudiantes del primer semestre de la Escuela de Enfermería. Decanato de Medicina. Universidad Centro occidental Lisandro Alvarado. Barquisimeto", en una muestra de 45 estudiantes, concluyó que el plan de afrontamiento al estrés no produjo cambios significativos, los estudiantes universitarios de la muestra estudiada reportaron como evento académico más estresante "presentar exámenes", los estudiantes utilizaron estrategias de afrontamiento al estrés más dirigidas al manejo emocional que al manejo del problema, se identificó inadecuadas estrategias de afrontamiento de estrés y hábitos de estudio inefectivos. En el Perú, 
Bustamante et $\mathrm{al}^{16}$, realizaron una investigación sobre "Ansiedad y estrés académicos en estudiantes de medicina Humana del $1^{\circ}$ y $6^{\circ}$ de la Universidad Mayor de San Marcos"; en una muestra de 98 estudiantes; concluyendo que existen mayores niveles de ansiedad en alumnos de primer año que en alumnos de sexto año, siendo estos resultados significativos, los principales generadores de estrés fueron la "sobrecarga académica", la "falta de tiempo para cumplir las actividades académicas" y la "realización de un examen".

Sánchez et $\mathrm{al}^{19}$ realizaron una investigación sobre "Estrés académico en estudiantes de Medicina de la Universidad Nacional de Trujillo" con el objetivo de determinar las características del estrés académico; mediante un estudio descriptivo relacional en una muestra de 159 alumnos de primero, cuarto y séptimo año concluyendo que: el nivel de estrés fue medianamente alto, los estresores predominantes fueron sobrecarga de tareas $26 \%$, evaluaciones $25 \%$ y exceso de responsabilidad $17 \%$, las estrategias de afrontamiento más utilizados fueron los esfuerzos por razonar y mantener la calma con un $26 \%$.

Alterio et $\mathrm{al}^{24}$ en Venezuela, realizaron una investigación sobre "Niveles de estrés, factores condicionantes y estrategias de afrontamiento en los estudiantes de post grado. Decanato de Medicina. Universidad Centro occidental Lisandro Alvarado. Barquisimeto, Estado Lara", con el objetivo de determinar los niveles de estrés, factores condicionantes y estrategias de afrontamiento en los estudiantes de post grado, mediante un estudio de tipo descriptivo de corte transversal en una muestra de 250 estudiantes, concluyendo que: el $65.6 \%$ tuvo estrés moderado, $23.1 \%$ estrés alto y $11.3 \%$ estrés bajo, las técnicas de afrontamiento más utilizadas fueron dormir y tomar café, y la menos utilizada fue fumar.

Sumado a esto existen otros eventos como lo social: disgusto con los miembros de la familia y amigos, malas relaciones interpersonales, conflictos religiosos, etc.; dinámica familiar: divorcio de los padres y exceso de exigencias de los mismos; económico: insuficiente salario de los padres o del propio estudiante que trabaja, finalmente en aspecto personal como la baja autoestima, cambio en los patrones de vida, el explorar reiteradamente múltiples áreas de estudio y trabajo. Hernández $\mathrm{H}$. et $\mathrm{al}^{25}$, México, al estudiar sobre "Factores determinantes de estrés y su asociación a toxicomanías en universitarios de enfermería de la Universidad Autónoma del Estado de México", con el objetivo de identificar los niveles de estrés y su asociación con toxicomanías en universitarios, con un estudio de tipo en una muestra de 327 alumnos, concluyeron lo siguiente: El nivel de estrés moderado (según la escala de Holmes y Rahe predispone a las toxicomanías y el riesgo se incrementa cuanto mayor sea el nivel de estrés (>500 UCV), las fuentes de estrés más frecuentes fueron el medio familiar $\mathrm{y}$ conductas de género.

Es así como, el estudiante modifica los efectos perjudiciales de los estresores mediante estrategias de afrontamiento o habilidades que desarrollan pudiendo ser directos 0 de evitación, con el fin de solucionar sus problemas. Berrrios ${ }^{26}$ realizó una investigación sobre "Nivel de vulnerabilidad frente al estrés y mecanismos de afrontamiento en estudiantes de Enfermería de la U.N.M.S.M." con el objetivo de determinar el nivel de vulnerabilidad frente al estrés y los mecanismos de afrontamiento que emplean los estudiantes; con un estudio descriptivo de corte transversal, en una muestra de 130 estudiantes; concluyendo lo siguiente: La vulnerabilidad frente al estrés se encuentra en un nivel medio con tendencia a alto, siendo el indicador más preponderante el hecho de conformarse con facilidad, el realizar ejercicios y tener amigos, y la estrategia de afrontamiento es la acción positiva hedonista $(41 \%)$ destacando la actitud de ignorar el problema, fijarse en lo positivo, seguida de una acción positiva esforzada (36\%). 
Por lo tanto seguir una carrera universitaria significa tener propensión a desarrollar algún nivel de estrés desencadenando problemas emocionales, cognitivos y fisiológicos. En Colombia, Astudillo, $\mathrm{C}^{27}$ realizó un estudio sobre "Efectos biopsicosociales del estrés en estudiantes de la Pontificia Universidad Javeriana de Cali”, con el objetivo de determinar los efectos biopsicosociales que produce el estrés en los estudiantes; el método fue descriptivo exploratorio transversal; la muestra estuvo conformada por 50 estudiantes; y finalmente concluyeron que hay gran influencia del estrés en el aspecto biológico, psicológico y social afectando directamente este equilibrio, como una consecuencia de factores externos al organismo.

En Chile, Huaquin ${ }^{13}$ al estudiar sobre "Exigencias académicas y estrés en las carreras de la Facultad de Medicina de la Universidad Austral" con el objetivo de determinar los niveles de exigencias académicas y sus consecuencias estresoras con un estudio de tipo descriptivo correlacional y de corte transversal; en una muestra de 225 estudiantes, concluyendo lo siguiente: Las carreras de la Facultad de Medicina muestran niveles de exigencias altos acompañados de niveles de estrés moderadamente altos; dentro de las carreras que comprende la Facultad de Medicina los estudiantes de la carrera de Enfermería fueron los que experimentaron mayores niveles de estrés con un $71 \%$ seguido de Medicina con un $65 \%$.

La enfermera, junto al equipo de profesionales de salud, debe detectar las necesidades de salud no sólo en el hogar, colegio, sino también en las universidades, en relación a las estrategias de afrontamiento de las situaciones de estrés que podrían afectar directa o indirectamente al bienestar biopsicosocial de esta población ${ }^{18}$.

Teniendo en cuenta la problemática anteriormente descrita surge la siguiente interrogante:

\section{¿Cuál es la relación entre el nivel de estrés y las estrategias de afrontamiento en los estudiantes de la Facultad de Enfermería-UNSA 2006?}

\section{OBJETIVOS}

\section{OBJETIVO GENERAL}

Determinar la relación entre el nivel de estrés y las estrategias de afrontamiento en estudiantes de la Facultad de Enfermería-UNSA 2006.

\section{OBJETIVOS ESPECÍFICOS}

- Caracterizar a la población de estudio por: edad, sexo, grado de estudio y condición de matrícula y su relación con el nivel de estrés.

- Determinar el nivel de estrés en los estudiantes.

- Identificar las estrategias de afrontamiento: Enfocados al problema, a la emoción y la percepción.

\section{HIPÓTESIS}

Existe relación entre el nivel de estrés y las estrategias de afrontamiento en los estudiantes de la Facultad de Enfermería-UNSA. Arequipa. 2006. 


\section{METODOLOGÍA}

\section{TIPO Y DISEÑO DE ESTUDIO}

El presente trabajo de investigación según Hernández ${ }^{28}$ es de tipo transeccional, con un diseño de correlación, por que se describen las variables en un solo momento y se analiza la correlación del nivel de estrés con la variable estrategias de afrontamiento.

\section{PROCEDIMIENTO}

1. Selección de las unidades de observación mediante muestreo probabilístico estratificado: La que estuvo conformada por 234 estudiantes de primero a cuarto año de la Facultad de Enfermería 2006.

2. Aplicación de la prueba piloto al $10 \%$ de la población en estudio.

3. Aplicación de los instrumentos en la población de estudio para la recolección de datos: Se utilizaron los instrumentos en relación a las variables:

Escala de estrés de Holmes y Rahe

Escala de afrontamiento de COPE.

4. Procesamiento y obtención de datos:

Los datos una vez obtenidos fueron vaciados en una base de datos del programa Excel, para el procesamiento estadístico se hizo uso del programa SPSS versión 11.01

5. Análisis e interpretación de resultados:

Se utilizaron las siguientes técnicas estadísticas:

- Técnicas de estadística descriptiva Frecuencias Absolutas Frecuencias Relativas

- Técnicas de estadística Inferencial Análisis de Varianza Prueba F: para comparar más de 3 opciones o valores

\section{POBLACIÓN Y MUESTRA}

La población sujeto de estudio estuvo conformada por los estudiantes de la Facultad de Enfermería del primer al cuarto año la misma que estuvo comprendida por 394 alumnos.

La muestra estuvo conformada por 234 alumnos mediante un muestreo probabilistico estratificado.

Fórmula: $\quad n=\frac{y^{2} \times p \times q \times N}{E^{2}(N-1)+y^{2} \times p \times q}$

$n=$ Tamaño de la muestra

$y^{2}=$ Nivel de confianza elegida

$p=$ Probabilidad de ocurrencia

$q=$ Probabilidad de no ocurrencia

$E=$ Error admisible 
Muestreo estratificado, fue hallado de acuerdo al porcentaje que representa cada año de estudio en relación a la población total, para luego ser aplicada a la muestra.

\begin{tabular}{|c|c|c|}
\hline Año de estudio & $\begin{array}{c}\text { No de } \\
\text { alumnos }\end{array}$ & $\begin{array}{c}\text { Porcentaje } \\
\text { (\%) }\end{array}$ \\
\hline Primero & 77 & 33 \\
\hline Segundo & 58 & 25 \\
\hline Tercero & 58 & 25 \\
\hline Cuarto & 41 & 17 \\
\hline TOTAL & 234 & 100 \\
\hline
\end{tabular}

\section{Criterios de inclusión}

- Estudiantes de enfermería matriculados del primer al cuarto año.

\section{Criterios de exclusión}

- Negatividad de los estudiantes.

\section{MÉTODO, TÉCNICAS E INSTRUMENTO DE RECOLECCIÓN DE DATOS}

Para la recolección de datos, se utilizó como método la encuesta, como técnica el cuestionario y como instrumentos: Escala de estrés de Holmes y Rahe y Escala de Estimación de Afrontamiento de COPE.

\section{Escala de estrés de Holmes y Rahe:}

En 1967, Holmes y Rahe inventaron una escala de estimación del reajuste social a través del cual media el estrés. La cual fue adaptada a la población de estudiantes, siendo modificada y aprobada por el psicólogo clínico del "Centro de Salud Mental Moisés Heresi", Doctor Ernesto Cazorla. La escala consta de 40 acontecimientos o sucesos vitales acaecidos en el último año, a los cuales se les asigna valores (UCV) relacionados con la cantidad de estrés, de tal manera que relaciona la acumulación de los cambios de vida que totaliza el estrés con la probabilidad de presentar manifestaciones psicosomáticas. De acuerdo a los puntajes obtenidos se presenta los siguientes niveles de estrés.

- Estrés Normal: Estrés dentro de los límites normales, cuando existe el $0 \%$ de posibilidad de presentar manifestaciones psicosomáticas. Va de 0 a 149 UCV

- Estrés Leve: Cuando existe el $33 \%$ de posibilidad de presentar manifestaciones psicosomáticas. Va de 150 a 199 UCV

- Estrés Moderado: Cuando existe el 50\% de posibilidad de presentar manifestaciones psicosomáticas. Va de de 200 a 299 UCV

- Estrés Severo: Cuando existe el $80 \%$ de posibilidad de presentar manifestaciones psicosomáticas. Va de de 300 a más UCV

\section{Escala de estimación de Afrontamiento de COPE :}

Es un instrumento desarrollado en los Estados Unidos de Norteamérica por Carver et al. ${ }^{30}$ en 1989, en una muestra de 978 estudiantes de la universidad de Miami.

En el Perú el cuestionario de "Afrontamiento disposicional" fue traducido y validado por Salazar ${ }^{31}$ en 1992, la versión traducida del cuestionario en primer lugar fue sometido al juicio de cuatro jueces psicólogos de profesión con conocimiento del idioma inglés, para que 
verifique su correcta traducción y la validez del contenido de cada una de las escalas, las observaciones de esto fueron tomados en cuenta para reemplazar algunos términos empleados en la traducción inicial. Posteriormente el instrumento fue aplicado a un grupo de 82 estudiantes universitarios de ambos sexos, que pertenecían a la "Pontificia Universidad Católica de Lima" y "Universidad Mayor de San Marcos"

El instrumento pretende evaluar las diferentes estrategias de afrontamiento que las personas utilizan frente a situaciones estresantes. La calificación de las respuestas se realiza en base al puntaje obtenido en la estrategia de acuerdo a las áreas que consta el cuestionario haciendo uso de una planilla y un instructivo de calificación. Los puntajes a alcanzar según el área de enfoque pueden ser:

- Estrategias de afrontamiento enfocados al problema; de 20 a 80

- Estrategias de afrontamiento enfocados a la emoción; de 20 a 80

- Estrategias de afrontamiento enfocados a la percepción; de 12 a 48

\section{ANÁLISIS Y DISCUSIÓN DE LOS RESULTADOS}

A continuación presentaremos los resultados obtenidos durante la investigación, los datos obtenidos se presentan en cuadros y gráfico de la siguiente manera:

- Información general: Tabla I

- Población estudio según estrés: Gráfico 1 y Cuadro 1

- Población de estudio según estrategias de afrontamiento: Cuadros 2, 3, 4 y 5

- Comprobación de hipótesis: Cuadro 6, 7, 8 y 9. TABLA I.- CARACTERIZACIÓN DE LA POBLACIÓN EN ESTUDIO SEGÚN NIVELES DE
ESTRÉS FACULTAD DE ENFERMERÍA - UNAS AREQUIPA - 2006

\begin{tabular}{|c|c|c|c|c|c|c|c|c|c|c|}
\hline \multirow{3}{*}{$\begin{array}{c}\text { GRUPO DE } \\
\text { EDAD }\end{array}$} & \multicolumn{8}{|c|}{ NIVEL DE ESTRÉS } & \multirow{2}{*}{\multicolumn{2}{|c|}{ TOTAL }} \\
\hline & \multicolumn{2}{|c|}{ Normal } & \multicolumn{2}{|c|}{ Leve } & \multicolumn{2}{|c|}{ Moderado } & \multicolumn{2}{|c|}{ Severo } & & \\
\hline & № & $\%$ & № & $\%$ & № & $\%$ & № & $\%$ & № & $\%$ \\
\hline 17 a 18 & 14 & 5.98 & 8 & 3.42 & 10 & 4.27 & 16 & 6.84 & 48 & $\begin{array}{c}20.5 \\
1\end{array}$ \\
\hline 19 a 20 & 27 & $\begin{array}{c}11.5 \\
4\end{array}$ & 22 & 9.40 & 25 & $\begin{array}{c}10.6 \\
8\end{array}$ & 32 & $\begin{array}{c}13.6 \\
8\end{array}$ & 106 & $\begin{array}{c}45.3 \\
0\end{array}$ \\
\hline 21 a 22 & 20 & 8.55 & 5 & 2.14 & 18 & 7.69 & 14 & 5.98 & 57 & $\begin{array}{c}24.3 \\
6\end{array}$ \\
\hline 23 a 24 & 4 & 1.71 & 1 & 0.43 & 6 & 2.56 & 4 & 1.71 & 15 & 6.41 \\
\hline $25 a+$ & 1 & 0.43 & 0 & 0.00 & 2 & 0.85 & 5 & 2.14 & 8 & 3.42 \\
\hline TOTAL & 66 & $\begin{array}{c}28.2 \\
1\end{array}$ & 36 & 15.38 & 61 & $\begin{array}{c}26.0 \\
7\end{array}$ & 71 & $\begin{array}{c}30.3 \\
4\end{array}$ & 234 & 100 \\
\hline SEXO & № & $\%$ & № & $\%$ & № & $\%$ & № & $\%$ & № & $\%$ \\
\hline Femenino & 60 & $\begin{array}{c}25.6 \\
4\end{array}$ & 34 & 14.53 & 52 & $\begin{array}{c}22.2 \\
3\end{array}$ & 66 & $\begin{array}{c}28.2 \\
0\end{array}$ & 212 & $\begin{array}{c}90.6 \\
0\end{array}$ \\
\hline Masculino & 3 & 1.28 & 2 & 0.85 & 6 & 2.56 & 11 & 4.71 & 22 & 9.40 \\
\hline TOTAL & 63 & $\begin{array}{c}26.9 \\
2\end{array}$ & 36 & 15.38 & 58 & $\begin{array}{c}24.7 \\
9\end{array}$ & 77 & $\begin{array}{c}32.9 \\
1\end{array}$ & 234 & 100 \\
\hline
\end{tabular}




\begin{tabular}{|c|c|c|c|c|c|c|c|c|c|c|}
\hline $\begin{array}{c}\text { TIPO DE } \\
\text { MATRÍCULA }\end{array}$ & № & $\%$ & № & $\%$ & № & $\%$ & № & $\%$ & № & $\%$ \\
\hline Regular & 60 & $\begin{array}{c}25.6 \\
4\end{array}$ & 33 & 14.10 & 58 & $\begin{array}{c}24.7 \\
9\end{array}$ & 61 & $\begin{array}{c}26.0 \\
7\end{array}$ & 212 & $\begin{array}{c}90.6 \\
0\end{array}$ \\
\hline Irregular & 6 & 2.56 & 3 & 1.28 & 3 & 1.28 & 10 & 4.27 & 22 & 9.40 \\
\hline TOTAL & 66 & $\begin{array}{c}28.2 \\
1\end{array}$ & 36 & 15.38 & 61 & $\begin{array}{c}26.0 \\
7\end{array}$ & 71 & $\begin{array}{c}30.3 \\
4\end{array}$ & 234 & 100 \\
\hline $\begin{array}{c}\text { AÑO DE } \\
\text { ESTUDIOS }\end{array}$ & № & $\%$ & № & $\%$ & № & $\%$ & № & $\%$ & № & $\%$ \\
\hline Primero & 22 & 9.40 & 8 & 3.42 & 18 & 7.69 & 29 & $\begin{array}{c}12.3 \\
9\end{array}$ & 77 & $\begin{array}{c}32.9 \\
1\end{array}$ \\
\hline Segundo & 11 & 4.70 & 11 & 4.70 & 11 & 4.70 & 25 & $\begin{array}{c}10.6 \\
8\end{array}$ & 58 & $\begin{array}{c}24.7 \\
9\end{array}$ \\
\hline Tercero & 20 & 8.55 & 9 & 3.85 & 17 & 7.26 & 12 & 5.13 & 58 & $\begin{array}{c}24.7 \\
9\end{array}$ \\
\hline Cuarto & 13 & 5.56 & 8 & 3.42 & 15 & 6.41 & 5 & 2.14 & 41 & $\begin{array}{c}17.5 \\
2 \\
\end{array}$ \\
\hline TOTAL & 66 & $\begin{array}{c}28.2 \\
1\end{array}$ & 36 & 15.38 & 61 & $\begin{array}{c}26.0 \\
7\end{array}$ & 71 & $\begin{array}{c}30.3 \\
4\end{array}$ & 234 & 100 \\
\hline
\end{tabular}

El mayor porcentaje de la muestra en estudio se encuentra ubicada en el grupo de edad de 19 a 20 años presentando un nivel de estrés severo, con respecto al sexo, predomina el femenino con mayor frecuencia, siendo la razón de 9/1 con relación al sexo masculino; tendencia que se viene evidenciando desde hace muchos años en enfermería, posiblemente a que cultural o socialmente sea más aceptada en este rol que el masculino. Del total de la población en estudio, tanto el femenino como el masculino presentaron niveles de estrés severo.

Se observa además que el primer año presenta estrés severo en mayor porcentaje seguido de segundo, tercero y cuarto año, ahora bien el grupo de estudiantes que ingresa por primera vez a una institución universitaria y la complejidad del proceso de adaptación a dicho ambiente, hace que los estudiantes se encuentren con la necesidad de establecer un cambio de perspectiva en su comportamiento para adaptarse al nuevo entorno; sin embargo, una gran parte de estos estudiantes carecen de estrategias adecuadas para el manejo para las nuevas demandas.

La entrada a la universidad representa un conjunto de situaciones altamente estresantes, debido a que el individuo pueda experimentar una falta de control sobre el nuevo ambiente potencialmente generador de estrés ${ }^{12}$.

Del total de la muestra en estudio son de tipo de matricula regular, tanto los alumnos regulares e irregulares presentan niveles de estrés severo. 


\section{GRÁFICO 1 \\ POBLACIÓN EN ESTUDIO SEGÚN NIVEL DE ESTRÉS \\ FACULTAD DE ENFERMERÍA - UNSA \\ PORCENTAJE \\ AREQUIPA - 2006}

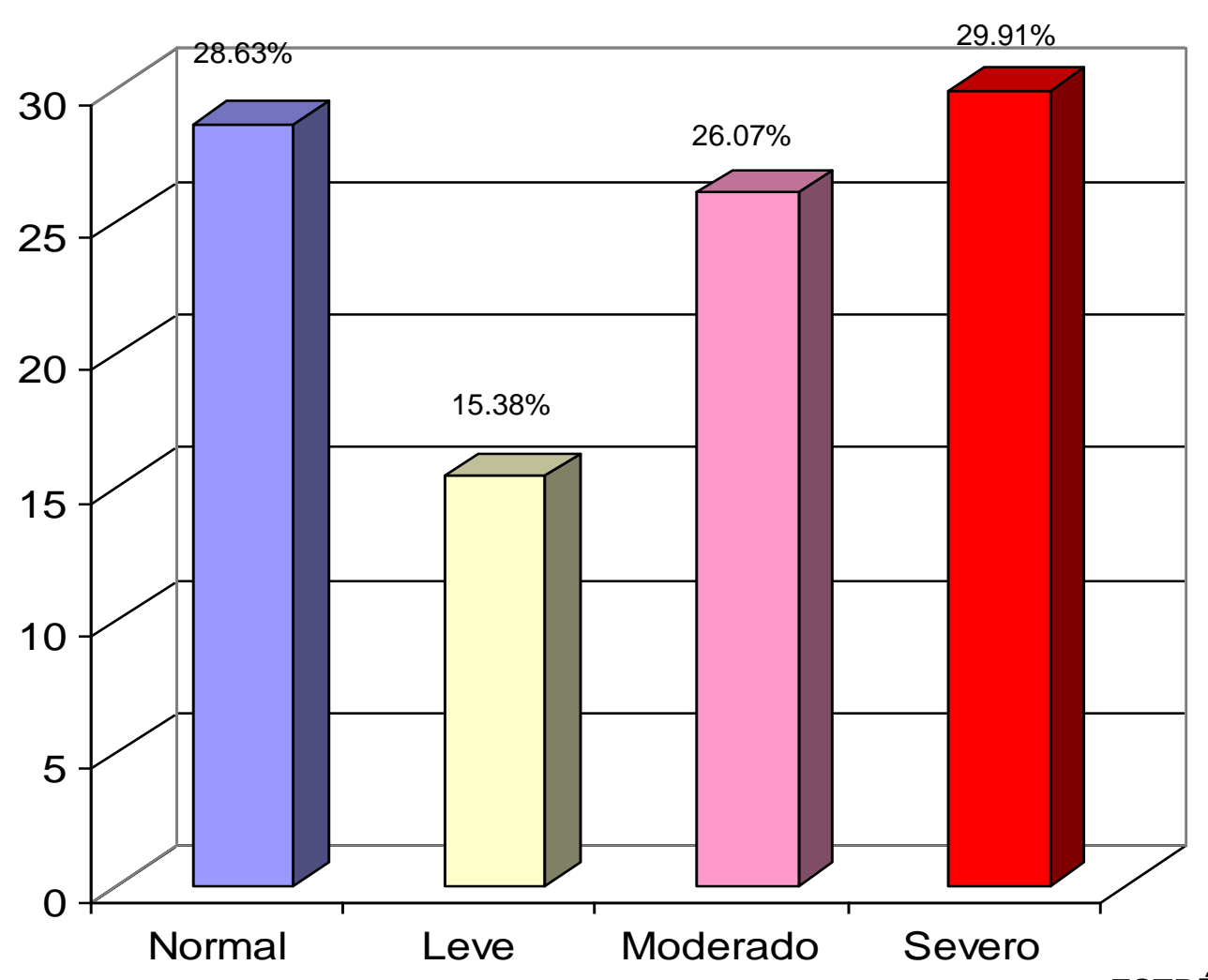

ESTRÉS

Del total de la muestra estudiada la mayoría presenta niveles de estrés severo; por lo cual inferimos que las demandas del medio son percibidas como excesiva, intensas, prolongadas y desagradables, que podrían superar la capacidad de resistencia del organismo llegando al distrés potencialmente perjudicial y nociva ${ }^{17}$, generando trastornos de salud derivados del estrés como trastornos digestivos, cardiovasculares, endocrinos e inmunológicos, entre otros.

Las investigaciones sobre el estudio y el afrontamiento del estrés evidencian el interés de distintos profesionales, poniendo de manifiesto que el siglo XXI es el siglo de los problemas de la humanidad derivados del estrés ${ }^{21}$.

\section{CUADRO 1.- POBLACIÓN EN ESTUDIO SEGÚN EVENTOS ESTRESANTES FACULTAD DE ENFERMERÍA - UNSA AREQUIPA - 2006}

\begin{tabular}{l|c|c}
\multicolumn{1}{c|}{ EVENTOS DE VIDA } & \multicolumn{2}{c}{ FRECUENCIA } \\
\cline { 2 - 3 } & $\mathbf{N} \mathbf{0}$ & $\%$ \\
\hline Proximidad de exámenes finales & 158 & 67,52 \\
\hline $\begin{array}{l}\text { Cambio en los hábitos de sueño (dormir más o menos } \\
\text { horas) }\end{array}$ & 146 & 62,39 \\
\hline Comienzo o fin del semestre & 120 & 51,28 \\
\hline Cambio en los hábitos alimenticios & 105 & 44,87 \\
\hline Problemas de rendimiento de estudios & 82 & 35,04 \\
\hline Problema serio de salud de un familiar cercano & 78 & 33,33 \\
\hline
\end{tabular}




\begin{tabular}{l|c|c}
\hline Cambio en el estado económico (propio o de los padres) & 75 & 32,05 \\
\hline Navidad y/o vacaciones & 74 & 31,62 \\
\hline Reconciliación con la familia, novio(a) o enamorado(a) & 65 & 27,78 \\
\hline $\begin{array}{l}\text { Cambio en el número de discusiones con la pareja, padres o } \\
\text { amigos }\end{array}$ & 65 & 27,78 \\
\hline $\begin{array}{l}\text { Cambio en las responsabilidades personales (trabajar y } \\
\text { estudiar, etc.) }\end{array}$ & 60 & 25,64 \\
\hline Muerte de un familiar cercano & 59 & 25,21 \\
\hline Separación de tu familia o de tu pareja & 58 & 24,79 \\
\hline Deuda personal o familiar de poco valor & 55 & 23,50 \\
\hline $\begin{array}{l}\text { Cambio del tipo de estudio (del colegio o academia a la } \\
\text { universidad) }\end{array}$ & 54 & 23,08 \\
\hline $\begin{array}{l}\text { Cambio en las condiciones de vida (remodelación, visitas, } \\
\text { etc.) }\end{array}$ & 54 & 23,08 \\
\hline Cambio en actividades sociales (frecuencia de salidas) & 51 & 21,79 \\
\hline Cambio en la frecuencia de reuniones familiares & 51 & 21,79 \\
\hline Accidente lesión grave o enfermedad personal & 48 & 20,51 \\
\hline Inseguridad en la elección de la carrera a seguir & 43 & 18,38 \\
\hline Divorcio, separación o peleas constantes de los padres & 41 & 17,52 \\
\hline $\begin{array}{l}\text { Cambio en hábitos personales (comenzar o dejar de fumar, } \\
\text { etc.) }\end{array}$ & 38 & 16,24 \\
\hline Logro personal sobresaliente (premios, etc.) & 36 & 15,38 \\
\hline Hipoteca o deuda personal o familiar de mucho valor & 34 & 14,53 \\
\hline Llegada de un nuevo miembro de la familia (nace un & 32 & 13,68 \\
\hline hermano, etc.) & 30 & 12,82 \\
\hline Problemas con profesores & 29 & 12,39 \\
\hline Problemas con parientes políticos o familia de la pareja & 23 & 9,83 \\
\hline Mudanza o cambio de residencia & 19 & 8,12 \\
\hline Cambio de carrera profesional & 16 & 6,84 \\
\hline Hermano(a) que abandona la casa & 16 & 6,84 \\
\hline Cambio de religión & 13 & 5,56 \\
\hline Muerte de los padres, novio(a) o enamorado(a) & 11 & 4,70 \\
\hline Embarazo indeseado (o de tu pareja) & 11 & 4,70 \\
\hline Muerte de un amigo íntimo & 8 & 3,42 \\
\hline Despido de un trabajo o ser expulsado de la universidad & 8 & 3,42 \\
\hline Cierre de una hipoteca o préstamo & 7 & 2,99 \\
\hline Compromiso de matrimonio & 4 & 1,71 \\
\hline Problemas de identidad sexual & 4 & 1,71 \\
\hline Detención en la cárcel o instituciones por violar la ley & 1,28 \\
\hline
\end{tabular}

Los cinco eventos más frecuentemente reportados por los estudiantes son la "Proximidad de exámenes finales", "Cambio en los hábitos de sueño", "Fin del semestre", "Cambio en los hábitos alimenticios" y finalmente "Problemas en el rendimiento de estudios" 
En cuanto a los eventos estresantes, llama la atención que los estudiantes mencionen como el de mayor relevancia la "proximidad de exámenes finales"; a diferencia del resto de eventos; el hecho de que el estudiante preste mayor atención al examen se debe a que en educación superior el peso de lo que un estudiante maneja en conocimiento descansa mayormente en la presentación de exámenes. Esta situación genera un grado de atención importante trayendo como consecuencias la modificación de sus estilos de vida según se acerca el período de exámenes, convirtiéndose así sus hábitos en insaludables, lo que, a la larga, puede llevar a la aparición de trastornos de salud como gastritis, cefaleas, etc.

Considerando a la enfermería en Salud mental según Evans ${ }^{29}$, "un área especializada de la práctica de la enfermería dirigida a tener un impacto tanto preventivo como correctivo sobre los trastornos mentales y sus secuelas, y se ocupa de la promoción de una óptima de la salud mental para la sociedad"

CUADRO 2.- PUNTAJES OBTENIDOS EN LA ESTRATEGIA DE AFRONTAMIENTO:
ENFOCADO AL PROBLEMA. FACULTAD DE ENFERMERÍA - UNSA AREQUIPA - 2006 ENFOCADO AL PROBLEMA. FACULTAD DE ENFERMERÍA - UNSA AR
PUNTAJES OBTENIDOS

\begin{tabular}{|c|c|c|c|c|c|c|}
\hline \multirow[b]{2}{*}{$\begin{array}{c}\text { ENFOCADO AL } \\
\text { PROBLEMA }\end{array}$} & \multirow[b]{2}{*}{$\begin{array}{l}\text { RANGO } \\
\text { TEÓRICO }\end{array}$} & \multicolumn{5}{|c|}{ PUNTAJES OBTENIDOS } \\
\hline & & Mínimo & Promedio & $\begin{array}{c}\% \\
\text { Prom. } \\
\left({ }^{*}\right)\end{array}$ & Máximo & $\begin{array}{l}\text { Desviación } \\
\text { Estándar }\end{array}$ \\
\hline $\begin{array}{l}\text { Afrontamiento } \\
\text { Activo }\end{array}$ & 4 a 16 & 5 & 9,79 & 61,19 & 16 & 2,06 \\
\hline Planificación & 4 a 16 & 5 & 10,93 & 68,31 & 16 & 2,10 \\
\hline $\begin{array}{l}\text { Supresión de } \\
\text { Actividades } \\
\text { Competentes }\end{array}$ & 4 a 16 & 4 & 8,81 & 55,06 & 16 & 1,92 \\
\hline Postergación & 4 a 16 & 4 & 8,87 & 55,44 & 15 & 1,76 \\
\hline $\begin{array}{c}\text { Búsqueda de } \\
\text { Apoyo Social por } \\
\text { Razones } \\
\text { Instrumentales }\end{array}$ & 4 a 16 & 4 & 9,67 & 60,44 & 16 & 2,24 \\
\hline
\end{tabular}

( $\left.{ }^{*}\right) \%$ Promedio $=$ Promedio $/$ máximo Teórico X 100

Con relación a las estrategias de afrontamiento enfocados al problema usados con mayor frecuencia son la "planificación" y el "afrontamiento activo", también se observa que la estrategia con menos frecuencia de uso es la "supresión de actividades competentes" y la "postergación".

Esto nos indica que los estudiantes "creen" que tiene posibilidades de modificar la situación por lo que hacen uso del planteamiento mental buscando las causas y las posibles soluciones para elegir la más conveniente, para luego ejecutar acciones que alteren la fuente del estrés con el fin de superar el problema, haciendo uso de un afrontamiento directo y conductual. 
CUADRO 3.- PUNTAJES OBTENIDOS EN LA ESTRATEGIA DE AFRONTAMIENTO: ENFOCADO A LA EMOCIÓN FACULTAD DE ENFERMERÍA - UNSA AREQUIPA - 2006

\begin{tabular}{|c|c|c|c|c|c|c|}
\hline \multirow[b]{2}{*}{$\begin{array}{c}\text { ENFOCADO A LA } \\
\text { EMOCIÓN }\end{array}$} & \multirow[b]{2}{*}{$\begin{array}{c}\text { RANGO } \\
\text { TEÓRICO }\end{array}$} & \multicolumn{5}{|c|}{ PUNTAJES OBTENIDOS } \\
\hline & & Mínimo & Promedio & $\begin{array}{c}\text { \% Prom. } \\
\left({ }^{*}\right)\end{array}$ & Máximo & $\begin{array}{c}\text { Desviación } \\
\text { Estándar }\end{array}$ \\
\hline $\begin{array}{l}\text { Búsqueda de } \\
\text { apoyo social por } \\
\text { razones } \\
\text { emocionales }\end{array}$ & 4 a 16 & 4 & 9,86 & 61,63 & 16 & 2,56 \\
\hline $\begin{array}{l}\text { Reinterpretación } \\
\text { positiva y } \\
\text { crecimiento }\end{array}$ & 4 a 16 & 4 & 10,64 & 66,50 & 16 & 2,23 \\
\hline Aceptación & 4 a 16 & 4 & 9,75 & 60,94 & 16 & 2,12 \\
\hline Negación & 4 a 16 & 4 & 10,52 & 65,75 & 15 & 2,24 \\
\hline Acudir a la religión & 4 a 16 & 4 & 7,52 & 47,00 & 11 & 1,75 \\
\hline
\end{tabular}

$\left({ }^{*}\right) \%$ Promedio = Promedio / máximo Teórico X 100

Las estrategias de afrontamiento enfocados a la emoción usadas con mayor frecuencia son la "Reinterpretación positiva y crecimiento" y la "Negación"; también se observa que la estrategia usada con menos frecuencia es "acudir a la religión" y la "aceptación". Esto nos indica que los estudiantes en mayor porcentaje "ven" con esperanza y optimismo el problema sin lograr cambios en la situación misma de tal manera que los estudiantes buscan el lado positivo del problema para un aprendizaje a futuro. Otros estudiantes se rehúsan que la situación estresante existe "no sucede nada"; esta estrategia a largo plazo no funciona; asignándole connotaciones a la experiencia como aprendizaje a futuro buscando reducir los estresores y afrontando de forma efectiva el problema.

CUADRO 4.- PUNTAJES OBTENIDOS EN LA ESTRATEGIA DE AFRONTAMIENTO: ENFOCADO A LA PERCEPCIÓN.FACULTAD DE ENFERMERÍA - UNSA AREQUIPA 2006

\begin{tabular}{c|c|c|c|c|c|c}
\hline & \multicolumn{6}{|c}{ PUNTAJES OBTENIDOS } \\
\cline { 3 - 7 } $\begin{array}{c}\text { ENFOCADO A } \\
\text { LA }\end{array}$ & RANGO \\
PERCEPCIÓN & TEÓRICO & Mínimo & Promedio & $\begin{array}{c}\% \\
\text { Prom. } \\
\left({ }^{*}\right)\end{array}$ & Máximo & $\begin{array}{c}\text { Desviación } \\
\text { Estándar }\end{array}$ \\
\hline $\begin{array}{c}\text { Enfocar y liberar } \\
\text { emociones }\end{array}$ & 4 a 16 & 4 & 8,75 & 54,69 & 15 & 2,14 \\
\hline $\begin{array}{c}\text { Desentendimiento } \\
\text { conductual }\end{array}$ & 4 a 16 & 4 & 7,03 & 43,94 & 13 & 1,91 \\
\hline $\begin{array}{c}\text { Desentendimiento } \\
\text { mental }\end{array}$ & 4 a 16 & 4 & 8,89 & 55,56 & 15 & 2,06 \\
\hline
\end{tabular}

( ${ }^{*}$ ) \% Promedio = Promedio / máximo Teórico X 100

Las estrategias de afrontamiento enfocadas al área de la percepción, usadas con mayor frecuencia son el "desentendimiento mental" y el "enfocar y liberar emociones", también se observa que la estrategia menos utilizada es el "desentendimiento conductual". Esto indica que los estudiantes permiten que una amplia variedad de actividad de actividades distraigan su evaluación en la dimensión conductual del problema por consiguiente interfieren con su meta. Otros estudiantes se enfocan en reacciones emocionales que experimentan y las expresan en forma abierta, y una menor frecuencia de estudiantes disminuyen sus esfuerzos y se dan por vencidos. 
CUADRO 5.- PUNTAJES PROMEDIOS OBTENIDOS EN LAS ESTRATEGIAS DE AFRONTAMIENTO FACULTAD DE ENFERMERÍA - UNSA AREQUIPA - 2006

\begin{tabular}{c|c|c|c|c|c|c}
\hline & \multicolumn{5}{|c}{ PUNTAJES OBTENIDOS } \\
\cline { 5 - 7 } AFRONTAMIENTO & $\begin{array}{c}\text { RANGO } \\
\text { TEÓRICO }\end{array}$ & Mínimo & Promedio & $\begin{array}{c}\% \\
\text { Prom. } \\
(*)\end{array}$ & Máximo & $\begin{array}{c}\text { Desviación } \\
\text { Estándar }\end{array}$ \\
\hline Al Problema & 20 a 80 & 23 & 48,08 & 60,10 & 79 & 7,32 \\
\hline A la Emoción & 20 a 80 & 23 & 48,31 & 60,39 & 70 & 2,10 \\
\hline A la Percepción & 12 a 48 & 12 & 24,67 & 51,40 & 37 & 4,32 \\
\hline
\end{tabular}

$\left({ }^{*}\right)$ \% Promedio = Promedio / máximo Teórico X 100

En lo que se refiere a las estrategias de afrontamiento, los estudiantes mostraron, una mayor tendencia hacia el manejo emocional y menor hacia el manejo del problema y por último a la percepción, esto indica que los estudiantes de la muestra estudiada manejan principalmente las emociones y actúan muy poco sobre el problema en sí. Estos resultados son similares con los hallazgos de otros autores, quienes encontraron que cuando los estudiantes creen tener poco control sobre las contingencias, promueven estilos de afrontamiento más dirigidos a la reducción de las emociones negativas que al manejo del problema ${ }^{19,20,24}$.

CUADRO 6.- RELACIÓN ENTRE NIVEL DE ESTRÉS Y ESTRATEGIA DE AFRONTAMIENTO ENFOCADO AL PROBLEMA FACULTAD DE ENFERMERÍA - UNSA AREQUIPA - 2006

\begin{tabular}{c|c|c|c|c|c}
\hline \multirow{2}{*}{$\begin{array}{c}\text { NIVEL DE } \\
\text { ESTRÉS }\end{array}$} & \multicolumn{5}{|c}{ PUNTAJES OBTENIDOS (20-80) } \\
\cline { 2 - 6 } & Mínimo & Promedio & $\begin{array}{c}\text { \% } \\
\text { Prom. }\end{array}$ & Máximo & $\begin{array}{c}\text { Desviación } \\
\text { Estándar }\end{array}$ \\
\hline Normal & 23 & 46,16 & 57,71 & 62 & 7,18 \\
\hline Leve & 37 & 49,14 & 61,42 & 79 & 9,32 \\
\hline Moderado & 36 & 47,90 & 59,88 & 61 & 5,74 \\
\hline Severo & 32 & 49,53 & 61,91 & 72 & 7,26 \\
\hline
\end{tabular}

Estadístico $\mathrm{F}=2.76$

Nivel de Significancia $=0.0427(E S)$

El nivel de estrés se relaciona de manera significativa con la estrategia de afrontamiento enfocada al problema; es decir, que a mayores niveles de estrés, los estudiantes hacen mayor uso de estrategias enfocadas al problema, las cuales son adecuadas y consideradas como exitosas ya que trabajan directamente con la situación estresante de modo que ésta se reduzca o desaparezca.

Sin duda, las estrategias enfocadas al problema son mas adecuadas y aconsejables por lo tanto debe practicarse y tratar de que sea la primera respuesta espontánea a situaciones estresantes, ya que implica no solo el manejo directo del problema sino del control de las emociones negativas generadas por el estrés. 
CUADRO 7.- RELACIÓN ENTRE NIVEL DE ESTRÉS Y ESTRATEGIA DE AFRONTAMIENTO ENFOCADO A LA EMOCIÓN FACULTAD DE ENFERMERÍA - UNSA AREQUIPA - 2006

\begin{tabular}{c|c|c|c|c|c}
\hline \multirow{2}{*}{$\begin{array}{c}\text { NIVEL DE } \\
\text { ESTRÉS }\end{array}$} & \multicolumn{5}{|c}{ PUNTAJES OBTENIDOS (20-80) } \\
\cline { 2 - 6 } & Mínimo & Promedio & $\begin{array}{c}\% \\
\text { Prom. }\end{array}$ & Máximo & $\begin{array}{c}\text { Desviación } \\
\text { Estándar }\end{array}$ \\
\hline Normal & 23 & 46,19 & 57,74 & 61 & 7,08 \\
\hline Leve & 29 & 49,36 & 61,70 & 70 & 7,61 \\
\hline Moderado & 28 & 48,84 & 61,05 & 65 & 8,37 \\
\hline Severo & 35 & 49,33 & 61,66 & 63 & 6,28 \\
\hline
\end{tabular}

Estadístico $\mathrm{F}=2.68 \quad$ Nivel de Significancia $=0.0473(\mathrm{ES})$

El nivel de estrés se relaciona de manera significativa con el uso de las estrategias orientadas a las emociones; es decir que los estudiantes se centran en los sentimientos provocados por los sucesos estresantes, las cuales están dirigidas a disminuir o manejar la perturbación emocional generada por la fuente de estrés, sin lograr cambios en la situación misma; esta actitud no es adecuada por que dejan para mañana o pasado mañana el problema que les preocupa y de acuerdo con investigaciones se ha comprobado que existe una correlación positiva entre el burnout y la tendencia a reaccionar mediante las emociones ${ }^{14,24}$.

CUADRO 8.- RELACIÓN ENTRE NIVEL DE ESTRÉS Y ESTRATEGIA DE AFRONTAMIENTO ENFOCADO A LA PERCEPCIÓN FACULTAD DE ENFERMERÍA UNSA AREQUIPA - 2006

\begin{tabular}{c|c|c|c|c|c}
\hline \multirow{2}{*}{ Nivel de Estrés } & \multicolumn{5}{|c}{ Puntajes Obtenidos (12-48) } \\
\cline { 2 - 6 } & Mínimo & Promedio & $\begin{array}{c}\% \\
\text { Prom. }\end{array}$ & Máximo & DS \\
\hline Normal & 12 & 24,12 & 50,25 & 33 & 4,03 \\
\hline Leve & 17 & 24,86 & 51,79 & 37 & 4,87 \\
\hline Moderado & 15 & 24,77 & 51,60 & 37 & 4,49 \\
\hline Severo & 14 & 25,04 & 52,17 & 33 & 4,19 \\
\hline
\end{tabular}

$$
\text { Estadístico F }=0.57 \quad \text { Nivel de Significancia }=0.6368(\mathrm{NS})
$$

El nivel de estrés no se relaciona de manera significativa con el uso de estrategias enfocadas al área de la percepción a diferencia de las otras áreas; es decir a mayor nivel de estrés el estudiante de enfermería no trata de distraer su atención en otras actividades, no se da por vencido, ni se desespera, ni se echa a llorar; por lo cual no actúa de forma negativa (minimización) ni es inflexible para adaptarse a lo inesperado (hipervigilancia focalizada). 


\begin{tabular}{c|c|c|c}
\hline $\begin{array}{c}\text { NIVEL DE } \\
\text { ESTRÉS }\end{array}$ & $\begin{array}{c}\text { AL } \\
\text { PROBLEMA }\end{array}$ & $\begin{array}{c}\text { A LA } \\
\text { EMOCIÓN }\end{array}$ & $\begin{array}{c}\text { A LA } \\
\text { PERCEPCIÓN }\end{array}$ \\
\hline Normal & 57,71 & 57,74 & 50,25 \\
\hline Leve & 61,42 & 61,70 & 51,79 \\
\hline Moderado & 59,88 & 61,05 & 51,60 \\
\hline Severo & 61,91 & 61,66 & 52,17 \\
\hline
\end{tabular}

Con relación a la frecuencia con la cual se mencionaron las estrategias frente al manejo del estrés, se encontró que las estrategias de manejo al problema fueron más utilizadas que las del manejo centradas en la emoción cuando el nivel de estrés es severo. Cuando el estudiante percibe la situación como altamente estresante utiliza estrategias enfocadas al problema porque considera que las consecuencias de los eventos son amenazantes y dañinos, por lo tanto pone en marcha sus recursos y/o posibilidades para el manejo de la situación, es decir, que sus acciones van dirigidas a modificar el estresor, de esta manera hacen uso de un afrontamiento efectivo o exitoso.

Esto concuerda con estudios anteriores, realizados para determinar estrategias de afrontamiento al estrés en universitarios, encontrando que los estudiantes mostraron estilos de afrontamiento dirigidos al problema al enfrentar situaciones altamente estresantes ${ }^{19,26}$.

\section{CONCLUSIONES}

Las características personales de la población en estudio fueron: sexo, predominantemente el femenino, el grupo de edad más frecuente fue de 19 a 20, la gran mayoría registra matrícula regular y el año de estudio que apuntó mayor número de estudiantes y mayor nivel de estrés fue el primer año, seguido de segundo, tercero y cuarto año.

Del total de la muestra estudiada la mayoría presenta niveles de estrés severo; la fuente de estrés según el tema traumático fue el estrés académico, es así que el evento más frecuentemente reportado como estresante es la "proximidad de exámenes finales".

En lo que se refiere a las estrategias de afrontamiento, los estudiantes mostraron, una mayor tendencia hacia el manejo emocional y menor hacia el manejo del problema y por último a la percepción, siendo las estrategias más utilizadas la "Reinterpretación positiva y crecimiento" y "negación".

Las estrategias más utilizadas en el área enfocada al problema fueron la "Planificación" y el "afrontamiento activo", seguido de las estrategias más enfocada a la percepción que fueron: "Desentendimiento mental" y "enfocar y liberar emociones.

El nivel de estrés se relaciona de manera significativa con el uso de estrategias enfocadas al problema; sin embargo, el uso de las emociones también se relaciona de manera significativa con elevados niveles de estrés.

\section{RECOMENDACIONES}

Se hace necesario el desarrollo de un curso teórico práctico con un contenido curricular importante durante la formación de pre-grado de manera que los estudiantes reciban una 
formación básica de afrontamiento al estrés principalmente en los primeros años, donde también debería contemplarse en la formación continua el desarrollo de talleres de técnicas para el manejo de situaciones, técnicas de solución de problemas (toma de decisiones), técnicas para facilitar el cambio de conducta.

Plan de intervención para disminuir el estrés, en el que se incluyan la relajación, meditación, el ejercicio y la musicoterapia; de esta manera contrarrestar los factores estresantes del entorno que generan tensión y reducir el efecto real o potencial que podrían afectar el desenvolvimiento del estudiante, lo cual se llevaría a cabo mediante la prevención primaria, secundaria y terciaria.

Se recomienda hacer un estudio experimental posterior luego de haber implementado las dos recomendaciones anteriores para establecer si resultaron efectivas, al disminuir los niveles de estrés, utilizando los mismos instrumentos.

\section{BIBLIOGRAFÍA}

1. Smeltzer SC, Bare BG, Brunner \& Suddarth. Manual de Enfermería Médico Quirúrgico. Estrés y Adaptación 8aㅡ Edición. México. Interamericana. 1998. V.1

2. Papalia D, Wendkos S. Psicología. España. Mc Graw-Hill. 1995.

3. Mainieri R. El estrés. Disponible en: http://healthclub.fortunecity.com/hockey/91/estres.html

4. Mashal EJ. Emoción y motivación. Mc Graw-Hill. 1999.

5. Fontana P. Control del estrés. El manual Moderno. Mexico. 1998.

6. Ortiz P. Estrés y sufrimiento. UNMSM. Metacrowler. 1999.

7. Spielberger $\mathrm{CH}$. Tensión y Ansiedad. México. 1990.

8. Garcia y Martines. Reacciones psicológicas en pacientes somáticos. Madrid McGrawHill. 1999.

9. Zaldiva P.D. Conocimiento y dominio del estrés. La Habana, Cuba Editorial Científica. 1996

10. Bonilla L. El Estrés. Disponible en: http://www.monografias.com/trabajos14/estres/estres.shtml

11. Orlandini, A. El estrés qué es y cómo evitarlo. Argentina. Fondo de Cultura Económica. 1996.

12. Polo A, Hernandez J, Poza C. Evaluación del estrés académico en estudiantes universitarios. Universidad Autónoma de Madrid. Disponible en: http://www.uco.es/organiza/centros/educacion/paginas/temporal/Sapuco/articulo.pdf

13. Huaquín V. y Loaiza R. Exigencias Académicas y Estrés en las Carreras de la Facultad de Medicina de la Universidad Austral de Chile. Estudios pedagógicos, 2004, 30 pp. 3959.

14. Rodríguez G. Efectos de un plan de técnicas de estudio en los estudiantes del primer semestre de la escuela de Enfermería. Decanato de Medicina. Universidad Centro occidental Lisandro Alvarado. 1997. Barquisimeto. Disponible en: http://www.ucla.edu.ve

15. Barraza M., A. El estrés académico en los alumnos de postgrado de la Universidad Pedagógica de Durango, Guadalajara. 2003. Disponible en: http://www.psicologiacientifica.com/publicaciones/biblioteca/articulos/ar-barraza01.htm.

16. Universidad de Granada. El estrés. Disponible en: http://www.ugr.es/ ve/pdf/estres.pdf

17. Medspain. Estrés y Trabajo. Disponible en: http://www.medspain.com/n3 feb99/stress.htm

18. Marriner T. Modelos y Teorías de Enfermería, 5aㅡ Edición, Mosby/Doyma. 2002. 
19. Sanchez E et al. Estrés Académico en Estudiantes de Medicina. Universidad Nacional de Trujillo. Propuesta de Intervención Pedagógica I Congreso Peruano de Educación Médica. 2001.

20. Bustamante M, Celis J, Cabrera D, Cabrera M, Alarcón W, Monge E. Ansiedad y Estrés Académico en Estudiantes de Medicina Humana del Primer y Sexto Año UNMSM. 2000. Disponible en: http://sisbib.unmsm.edu.pe/Bvrevistas/Anales/v62 n1/Ansiedad.htm

21. Murray CJL e Lopez AD. The Global Burden of Diseases. WHO. 1990-2020. Nature America Inc. 1998; (11):1241-43.

22. Salud, Estrés y Factores Psicológicos. Disponible en: http://www.aidex.es/estres/articuloseloisa/eloisa2.htm

23. Olivares A. Conductas de afrontamiento al estrés en adolescentes pre-universitarios. Academias Fleming y Bardet. Arequipa. 1999.

24. Alterio G. y Cols. Niveles de estrés, factores condicionantes y estrategias de afrontamiento en los estudiantes de post grado. Decanato de Medicina. Universidad Centro occidental Lisandro Alvarado. Barquisimeto, Estado Lara. 2003. Disponible en: http://bibmed.ucla.edu.ve/

25. Hernández H. y Cols. Factores determinantes de estrés y su asociación a toxicomanías en universitarios de la universidad autónoma del estado de México. 2005. Disponible en: http://blog.pucp.edu.pe/item/9091

26. Berrrios F. Nivel de vulnerabilidad frente al estrés y mecanismos de afrontamiento en estudiantes de Enfermería de la U.N.M.S.M. 2005. Disponible en:

http://www.cybertesis.edu.pe/sdx/sisbib/imprimer.xsp?id=sisbib.2006.berrios gfprincipal\&partie=sisbib.2006.berrios gf $\mid \mathrm{TH} .5 \&$ base=documents

27. Astudillo C. y Avendaño C. Efectos Biopsicosociales del estrés en estudiantes de la Pontificia Universidad Javeriana de Cali. 2000. Disponible en: http: //www.mtas.es/insht/nto/ntp 439.htm

28. Hernández SR, Fernández C. Metodología de la investigación 3ª edición. Mc Graw-Hill. México 2003.

29. EEvans A, Gask L, Singleton C, Barhami J. Teaching consultation skills: a survey of general practice trainer. Medical Education. 2001. 35, p. 222-224

30. Carver, C. S., Scheier, M. F., Weintraub, J. K. Assessing coping strategies: A theoretically based approach. Journal of Personalityand Social Psychology. 1989. 56, p. 267-283.

31. Salazar, V. Relación entre los estilos de afrontamiento al estrés y las dimensiones de personalidad neurotisismo y extroversión. Estudio realizado en estudiantes universitarios. [Tesis de Licenciatura]. Lima: Universidad Peruana Cayetano Heredia. 1992. 